\title{
A SUBNORMAL SEMIGROUP WITHOUT NORMAL EXTENSION ${ }^{1}$
}

\author{
ARTHUR LUBIN
}

\begin{abstract}
TheOREM 1. There exists a subnormal semigroup with no commuting normal extension. THEOREM 2. There exist two commuting quasinormal operators without commuting normal extension.
\end{abstract}

1. A bounded linear operator $T$ on a Hilbert space $\mathcal{H}$ is called subnormal if there exists a normal operator $N$ acting on a larger Hilbert space $\mathscr{K} \supset \mathcal{H}$ such that $\mathcal{H}$ is invariant under $N$ and $\left.N\right|_{\mathcal{K}}=T$. T. Ito [3] showed that commuting subnormals $T_{1}, \ldots, T_{n}$ on $\mathcal{H}$ have a commuting normal extension, i.e. there exist commuting normals $N_{1}, \ldots, N_{n}$ each defined on $\mathcal{K} \supset \mathcal{H}$ with $\left.N_{i}\right|_{\mathcal{K}}=T_{i}$, if and only if an analog of the Halmos-Bram positivity condition is satisfied. Ito also showed that every continuous oneparameter semigroup of commuting subnormals on $\mathcal{H}$ can be extended to a continuous one-parameter semigroup of commuting normals on some $\mathcal{K} \supset$ $\mathcal{H}$. Recent examples by M. B. Abrahamse [1] and A. Lubin [4] show that there exist commuting subnormals with no commuting normal extensions. In this note we give an example of a two-parameter subnormal semigroup without commuting normal extension; our example also provides two commuting quasinormal operators without commuting normal extension. Our example is presented in a simplified form suggested by Professor Chandler Davis.

2. Let $\mathcal{H}$ be the Hilbert space having orthonormal basis $\left\{c_{0}, e_{n}, f_{n}: n=1\right.$, $2, \ldots\}$, and define $U_{1}, U_{2}$ on $\mathcal{H}$ by:

$$
\begin{aligned}
& U_{1}\left(e_{n}\right)=e_{n+1}, \\
& U_{1}\left(c_{0}\right)=e_{1}, \\
& U_{1}\left(f_{n}\right)=0, \\
& U_{2}\left(e_{n}\right)=0, \\
& U_{2}\left(c_{0}\right)=f_{1}, \\
& U_{2}\left(f_{n}\right)=f_{n+1},
\end{aligned}
$$

i.e.

Received by the editors September 17, 1976 and, in revised form, December 3, 1976.

AMS (MOS) subject classifications (1970). Primary 47B20; Secondary 47D05.

Key words and phrases. Subnormal operator, quasi-normal operator, commuting normal extension, subnormal semigroup.

${ }^{1}$ Research supported by NSF Grant MSC 76-06516. 


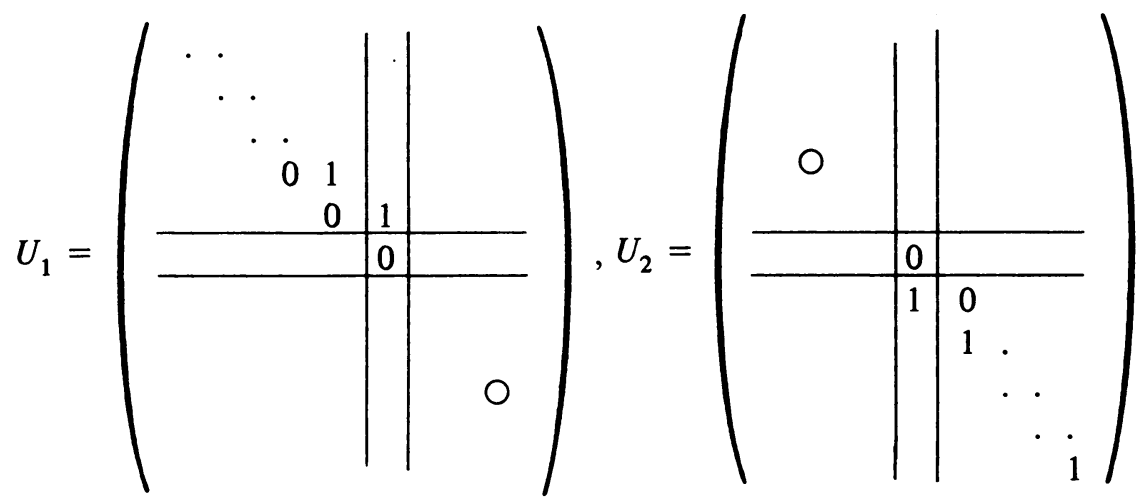

Note that each $U_{i}$ is the direct sum of a unilateral shift and 0 , and that $U_{1} U_{2}=U_{2} U_{1}=0 . \quad U_{1}$ and $U_{2}$ are clearly subnormal, and are even quasinormal. (Recall that an operator $A$ is quasinormal if $A\left(A^{*} A\right)=$ $\left(A^{*} A\right) A$ and every quasi-normal operator is subnormal $[2$, p. 101].) Since the powers of a subnormal are subnormal, $\delta=\left\{U_{1}^{j} U_{2}^{k}: j, k=0,1,2, \ldots\right\}$ is a commutative subnormal semigroup.

If there exists a commutative normal semigroup $\left\{N_{1}^{j} N_{2}^{k}\right\}$ on $\mathcal{K}$ extending $\delta$, then $\left(U_{1}+U_{2}\right)=\left.\left(N_{1}+N_{2}\right)\right|_{\mathcal{H}}$ is subnormal. An operator $A$ is called hyponormal if $\left(A^{*} A-A A^{*}\right) \geqslant 0$, and all subnormal operators are hyponormal [2, p. 103]. A simple computation shows that for $X=U_{1}+U_{2}$,

$$
Q=X^{*} X-X X^{*}=\left(\begin{array}{c|c|c}
0 & & \\
\hline-1 & & \\
\hline & & 0
\end{array}\right)
$$

Thus, $\left(Q\left(e_{1}+f_{1}\right),\left(e_{1}+f_{1}\right)\right)=-2<0$ so $Q$ is not even hyponormal and therefore $\delta$ cannot have a normal extension. Hence, we have

THEOREM 1. There exists a commuting subnormal semigroup with no commuting normal extension.

THEOREM 2. There exist two commuting quasinormal operators without a commuting normal extension.

Acknowledgement. This research was done at the Summer Research Institute in Operator Theory at the University of New Hampshire. The author is most grateful to the University and its staff for their gracious hospitality. 


\section{REFERENCES}

1. M. B. Abrahamse, Commuting subnormal operators, Illinois J. Math. (to appear).

2. P. R. Halmos, A Hilbert space problem book, Van Nostrand, Princeton, N.J., 1967. MR 34 \#8178.

3. T. Ito, On the commutative family of subnormal operators, J. Fac. Sci. Hokkaido Univ. Ser. I 14 (1958), 1-15.

4. A. Lubin, Weighted shifts and products of subnormal operators, Indiana Univ. Math. J. 26 (1977), 839-845.

Department of Mathematics, Illnnois Institute of Technology, Chicago, Illnois 60616 